\title{
Functional Disconnection of the Medial Prefrontal Cortex and Subthalamic Nucleus in Attentional Performance: Evidence for Corticosubthalamic Interaction
}

\author{
Yogita Chudasama, ${ }^{1}$ Christelle Baunez, ${ }^{2}$ and Trevor W. Robbins ${ }^{1}$ \\ ${ }^{1}$ Department of Experimental Psychology, University of Cambridge, Cambridge, CB2 3EB, United Kingdom, and ${ }^{2}$ Laboratoire de Neurobiologie de la \\ Cognition, Centre National de la Recherche Scientifique, 13402 Marseille cedex 20, France
}

\begin{abstract}
The present study used a disconnection procedure to test whether the medial prefrontal cortex (mPFC) and the subthalamic nucleus (STN) were functional components in a common neural system that controlled continuous performance in a test of sustained and divided visual attention. Animals with disconnected lesions of the MPFC and STN in contralateral hemispheres were severely impaired in several aspects of performance, including discriminative accuracy, increased perseveration, and slowed response latencies during postoperative testing. These deficits persisted throughout the entire experimental test period. Increasing the stimulus duration alleviated the accuracy deficit but failed to improve speed of responding or reduce perseverative responses. These deficits were in marked contrast to animals with combined mPFC and STN lesions made on the ipsilateral side, which produced behavior not different from that of sham controls. Rats with unilateral lesions of either structure alone were also impaired in terms of accuracy and perseveration. These data suggest that some of the deficits observed after bilateral STN lesions (attention and perseveration) may be attributable to a disrupted corticosubthalamic projection. This study provides direct evidence that performance in tasks that require optimal attentional and executive control relies on a corticosubthalamic interaction within the neural circuitry of the basal ganglia.
\end{abstract}

Key words: disconnection; attention; medial prefrontal cortex; subthalamic nucleus; behavior; basal ganglia

\section{Introduction}

A re-evaluation of the functional anatomy of the basal ganglia has highlighted the significance of a direct corticosubthalamic projection in influencing the activity of the basal ganglia (Bolam et al., 2002). Apart from the striatum, the subthalamic nucleus (STN) is the only structure in the basal ganglia to receive a direct cortical projection (Albin et al., 1989; Parent and Hazrati, 1995). This independent corticosubthalamic projection originates from the motor and premotor areas (Afsharpour, 1985; Canteras et al., 1990; Bolam et al., 2002) as well as the medial prefrontal cortex (mPFC), which includes the prelimbic and ventromedial prefrontal areas (Kitai and Deniau, 1981; Berendse and Groenewegen, 1991; Maurice et al., 1998). This direct interaction between the frontal cortex and the STN implicates its participation not only in sensorimotor functions but also in higher-order cognitive processing.

In line with this speculation, it has been shown that in rodents, bilateral lesions of the STN reverse motor impairments induced

Received Feb. 25, 2003; revised April 9, 2003; accepted April 9, 2003.

This work was supported by Centre National de la Recherche Scientifique (C.B.), Fifth Programme-Cadre de Recherche et de Développement Technologique of the European community (contract number QLK6-1999-02173) (C.B. and T.W.R.) with the Royal Society, and a programme grant from the Wellcome Trust (T.W.R.), completed within the Medical Research Centre for Behavioral and Clinical Neuroscience. Y.C. was supported by Cambridge Cognition. We thank Dr. K. Thomas for her help with photographic assistance.

Correspondence should be addressed to Dr. Yogita Chudasama, Laboratory of Neuropsychology, National Institute of Mental Health, 49 Convent Drive, Building 49, Room 1B80, Bethesda, MD 20892-4415. E-mail: yogita@In.nimh.nih.gov. Copyright $\odot 2003$ Society for Neuroscience $\quad 0270-6474 / 03 / 235477-09 \$ 15.00 / 0$ by dopamine depletion in a simple reaction time task while also producing nonmotor deficits such as premature responding, thus implicating a response control deficit (Baunez et al., 1995). STNlesioned rats also show multiple deficits in the five choice serial reaction time task (5CSRTT), including severe impairments in discriminative accuracy, perseveration, and premature responding (Baunez and Robbins, 1997). These deficits are also found after medial striatal lesions (Rogers et al., 2001), frontostriatal disconnections (Christakou et al., 2001), and bilateral mPFC lesions (Muir et al., 1996; Passetti et al., 2000). These data not only emphasize the influence of cortical projections on cognitive processing within the basal ganglia but also highlight the significance of corticosubthalamic interactions. Despite the controversy concerning the contralaterality of this projection or whether it is monosynaptic or polysynaptic (Parent and Hazrati, 1995), physiological studies have confirmed that stimulation of the contralateral prefrontal cortex evokes excitatory responses in the STN that are completely eliminated by a parasagittal knife cut of the corpus callosum (Fujimoto and Kita, 1993). Furthermore, excitation of the STN induced by contralateral prefrontal cortex stimulation is significantly evident even in ipsilaterally decorticated rats (Rouzaire-Dubois and Scarnati, 1985). These insights have encouraged a re-evaluation of the functional organization of the basal ganglia with a particular emphasis on the role of the corticosubthalamic projection (Berns and Sejnowski, 1996; Levy et al., 1997; Bolam et al., 2002).

The present study is the first to investigate the interdependent 
function of the MPFC and the STN in any behavioral setting (in this case, the 5CSRTT). Animals were first trained on this task and then prepared with either ipsilateral (same hemisphere) or disconnected (different hemisphere) lesions of the mPFC and STN. Baseline test sessions were followed by manipulations in stimulus duration and a test of spontaneous locomotor activity. It was predicted that if the $\mathrm{MPFC}$ and STN were functionally interdependent, the contralateral group would show profound impairments compared with the ipsilateral group. Rats with unilateral STN or unilateral mPFC lesions were also included for comparison.

\section{Materials and Methods}

Subjects. All subjects were male Lister Hooded rats (Charles River Laboratories, Kent, UK), housed in pairs in a temperature controlled room $\left(22^{\circ} \mathrm{C}\right)$, under diurnal conditions $(12 \mathrm{hr}$ light/dark cycle). Rats were deprived of food and maintained at $85 \%$ of their free-feeding weight throughout the experiment. All testing occurred at a regular time during the light period. Animals were 3 months of age and weighed $230-260 \mathrm{gm}$ at the start of behavioral training. All experimental procedures were subject to UK Home Office approval (Project License PPL 80/1324).

Apparatus. The test apparatus consisted of four $25 \times 25 \mathrm{~cm}$ aluminum chambers (Cambridge Cognition, Cambridge, UK). The rear wall of each chamber was concavely curved and contained nine apertures, each 2.5 $\mathrm{cm}$ square, $4 \mathrm{~cm}$ deep, and set $2 \mathrm{~cm}$ above floor level. Illumination of each hole was provided by a standard $3 \mathrm{~W}$ bulb located at the rear of the hole. In addition, an infrared photocell beam monitoring the nose poke responding of the rat was located at the entrance of each aperture. Each aperture could be blocked by a metal cover when not required, and for the present task, apertures 1, 3, 5, 7, and 9 were open (Carli et al., 1983).

The four chambers were individually housed within sound-attenuating cabinets and ventilated by low-level noise fans, which also served to mask extraneous background noise. The apparatus and online data collection were controlled by means of an Acorn computer system with software written by Dr. R. N. Cardinal (Cambridge University, Cambridge, UK).

Behavioral procedure. Rats were trained to discriminate a brief visual stimulus presented randomly in one of the five spatial locations (hole number 1, 3, 5, 7, and 9), as described previously (Muir et al., 1996; Baunez and Robbins, 1997). At the beginning of each test session, the house light was illuminated and free delivery of a single food pellet to the magazine was made. The trial was initiated by the rat opening the panel to collect this pellet. After a fixed $5 \mathrm{sec}$ intertrial interval (ITI), the light at the rear of one of the apertures was illuminated for a short period $(0.5$ $\mathrm{sec})$. Responses in this aperture during illumination and for $5 \mathrm{sec}$ afterward (the limited hold period) were rewarded with the delivery of a food pellet, and a correct response was recorded. Responses in a nonilluminated hole during the signal period (incorrect response) and failure to respond within the limited hold period (omission) were punished with a period of darkness during which all lights were extinguished for $5 \mathrm{sec}$ (time out). Responses in the apertures during the ITI were recorded as premature responses and punished with time out. Additional responses in the apertures during the limited hold period were recorded as perseverative responses. A response in the food panel after the delivery of a food pellet, or after the time-out period, initiated the next trial.

During any one session, the light stimulus was presented an equal number of times in each of the five apertures in a random order. A daily session consisted of 100 trials or was terminated after 30 min of testing. For the first session of training, the stimulus duration and limited hold periods were both set at $1 \mathrm{~min}$, and the ITI and time-out periods were set at $5 \mathrm{sec}$. These variables were altered on subsequent sessions according to the individual animal's performance, until the target set of task parameters could be instituted. The target parameters were as follows: stimulus duration, $0.5 \mathrm{sec}$; limited hold period, $5 \mathrm{sec}$; ITI and time-out period, 5 sec. The animals were considered to have reached criterion when these target parameters were attained on five consecutive sessions with $>80 \%$ correct responses and $<20 \%$ omissions within the $30 \mathrm{~min}$ session time. Approximately 30 sessions were required for the animals to attain this criterion.
Two weeks after surgery, the animals used in this experiment were tested across 12 sessions on the standard schedule of the task. To further investigate the observed deficits in accuracy, the attentional load was reduced by increasing the stimulus duration from 0.5 to 1,2 , and $4 \mathrm{sec}$ on separate days. Each day was preceded by a baseline session.

Performance measures. Accuracy of performance was measured as the proportion of correct responses (number of correct responses per total number of responses) expressed as a percentage. This measures errors of commission without including errors of omission. Errors of omission were also presented, because this measure reflects possible failures of detection and/or motivational-motor deficits, depending on the overall pattern of effects.

Speed, including decision time, was assessed according to two different latencies to the nearest centisecond. The first was the latency to respond correctly, defined as the time between the onset of the visual stimulus and the point at which the animal's nose breaks the infrared beam of the lit hole. The second measure was magazine latency (the time between performance of a correct response and the opening of the magazine panel to collect the food pellet).

The number of premature and perseverative responses was also recorded, as well as the number of perseverative panel pushes.

Locomotor activity. After postoperative testing, animals were placed in locomotor activity cages $(40 \times 25 \times 18 \mathrm{~cm})$ to assess the activity of the lesioned animals. The cages were equipped with infrared photocell beams that ran horizontally along the axis of the cage $(6 \mathrm{~cm}$ from the cage end and $1 \mathrm{~cm}$ above the floor). Beam interruptions were recorded over 10 min bins over a $2 \mathrm{hr}$ period. The apparatus and data collection were controlled by means of an Acorn computer system equipped with SPIDER extension (Paul Fray, Cambridge, UK) with software written by Dr. R. N. Cardinal.

Data analysis. Data for each variable were subjected to repeatedmeasures ANOVA using the SPSS statistical package, version 9.0 (SPSS, Chicago, IL). Data were initially explored using "boxplots" and tests of homogeneity of variance so that outliers were identified and so that skewed data, which violate the distribution requirement of the ANOVA, were transformed appropriately (arcsine, square root, or logarithmic), as recommended by Winer (1971). Homogeneity of variance across groups was assessed by the Mauchly sphericity test. When data sets significantly violated this requirement for a repeated-measures design, the HuynhFeldt $\epsilon$ was used to calculate a more conservative $p$ value for each $F$ ratio. Where $F$ ratios were significant, post hoc comparisons were made using Tukey's test. The criterion for statistical significance was $p<0.05$. The between-subject factor was lesion [three levels: sham controls, ipsilateral lesions (IPLs), and disconnected (contralateral) lesions (DISC)]. The within-subject factor included session (12 d) and the stimulus duration challenge as appropriate. The same statistical procedures were applied to data from the unilateral STN and unilateral mPFC groups.

Surgical procedures. Rats were divided into three groups. All animals were deeply anesthetized by intramuscular injections of xylazine $(10 \mathrm{mg} /$ $\mathrm{kg}$ ) and ketamine $(50 \mathrm{mg} / \mathrm{kg})$ and placed in a Kopf stereotaxic headholder (David Kopf Instruments, Tujanga, CA) fitted with atraumatic earbars. The scalp was retracted to expose the skull, and craniotomies were made directly above the target region of the brain. The incisor bar was set at $-3.0 \mathrm{~mm}$. Lesions were made using a $10 \mu \mathrm{l}$ Hamilton precision microsyringe mounted in a Harvard infusion pump (Harvard Apparatus, Kent, UK), connected via a fine bore polythene tubing $(0.28 \mathrm{~mm}$ outer diameter, $0.16 \mathrm{~mm}$ inner diameter; Portex, Kent, UK) to a 30 gauge stainless-steel cannula.

For lesions of the STN, the injection coordinates were taken as the average of interaural and bregma coordinates from Paxinos and Watson (1997). Infusions of $0.5 \mu \mathrm{l}$ of $0.06 \mathrm{M}$ ibotenic acid (Research Biochemicals, St. Albans, UK) dissolved in sterile PBS, pH 7.0-7.2, were made over $3 \mathrm{~min}$. The injector was left in place for an additional $5 \mathrm{~min}$ before being withdrawn. The injection coordinates for the STN lesions from bregma were as follows: anteroposterior (AP), $-3.8 \mathrm{~mm}$; lateral (L), $\pm 2.4 \mathrm{~mm}$; dorsoventral (DV), $-8.35 \mathrm{~mm}$ (from the skull). The coordinates from the interaural point were $\mathrm{AP},+5.2 \mathrm{~mm}$;, $\pm 2.4 \mathrm{~mm}$; and $\mathrm{DV},+1.65$ $\mathrm{mm}$.

For lesions of the mPFC, animals received four infusions of $0.5 \mu \mathrm{l}$ of 
$0.06 \mathrm{M}$ ibotenic acid at the following coordinates from bregma: $\mathrm{AP},+3.8$ $\mathrm{mm} ; \mathrm{L}, \pm 0.7 ; \mathrm{DV},-3.0 \mathrm{~mm}$; AP, $+3.2 \mathrm{~mm} ; \mathrm{L}, \pm 0.7 ; \mathrm{DV},-3.0 \mathrm{~mm}$ and $-2.0 \mathrm{~mm}$; and AP, $+2.7 \mathrm{~mm}$; L, $\pm 0.7 \mathrm{~mm}$; DV, $-3.0 \mathrm{~mm}$. All DV coordinates were taken from dura. Each injection was made over 3 min and allowed to remain for an additional $2 \mathrm{~min}$ for dispersion.

A total of 26 rats were used for the disconnection study. Ten animals received combined contralateral DISC. Eight animals received IPLs of the mPFC and STN. The side on which the lesion was made (right or left hemisphere) was counterbalanced for each group. An additional eight animals served as sham controls: four of these animals were contralateral, and four were ipsilateral. These animals received the same surgical treatment, except that they were infused with vehicle. An additional 28 animals received unilateral STN lesions $(n=10)$ or unilateral mPFC lesions $(n=10)$ or served as sham controls $(n=8)$. The side of the lesion was counterbalanced across group.

Histology. At the conclusion of the behavioral testing, the animals were perfused transcardially with $0.9 \%$ saline followed by $10 \%$ formal saline. After dehydration by immersion in $20 \%$ sucrose, brains were sectioned on a freezing microtome at $60 \mu \mathrm{m}$ thickness. Every second section was mounted on glass slides and stained with cresyl violet. The sections were used to verify lesion placement and to assess the extent of lesion-induced neuronal loss.

\section{Results}

\section{Histological analysis}

The cytoarchitectonic borders and nomenclature were taken from the atlas by Paxinos and Watson (1997). The extent of the lesions is illustrated in Figure 1. Animals with lesions made to the mPFC showed extensive cell damage, which began at the frontal pole and included the most rostral medial and orbital regions. The lesion began at bregma +4.2 and extended caudally to bregma +2.2 . The lesion included damage to the prelimbic cortex, infralimbic cortex, and in most cases the most ventral limits of the dorsal peduncular cortex regions (Fig. 1). Gliosis was also observed in the pregenual anterior cingulate cortex $(\mathrm{Cg} 1)$ at the most rostral level, although in most cases, the Cg1 was spared as the lesion extended caudally. In four animals of this group, the lesion encroached postgenu into the rostral limits of the anterior cingulate (Cg2).

STN lesions consistently involved the entire extent of the STN and induced shrinkage of the structure. The cell loss in the STN began at bregma -3.6 and extended caudally to bregma -4.3 . Within this group, four animals had cell damage that was centered on the most medial portion of the STN. Despite the close proximity of the medial STN to the adjacent lateral hypothalamus, there was no evidence of damage to the lateral hypothalamic region in this group of animals. One animal had a lesion that extended laterally into the most ventral limits of the zona incerta. In addition, calcium deposits were observed in the entopeduncular nucleus (seven animals) and within the STN region (two animals) (Baunez and Robbins, 1997). For the unilateral lesioned groups, three animals with mPFC lesions showed incomplete lesions with no significant neuron loss. One animal showed extensive bilateral damage. These animals were discarded from analysis. The remaining six animals had mPFC lesions that were centered on the prelimbic area (PrL) and extended caudally to the most rostral limits of the anterior cingulate cortex postgenu. In the unilateral STN group, all showed calcification in the entopeduncular nucleus and cell loss that was centered on the medial extent of the nucleus. In this group, two animals were found to have no lesion and another two animals showed damage that encroached into the substantia nigra. These animals were discarded from analysis.

The final numbers for the disconnection analysis were as follows: sham, $n=8$; IPL, $n=8$; DISC, $n=10$. The final numbers

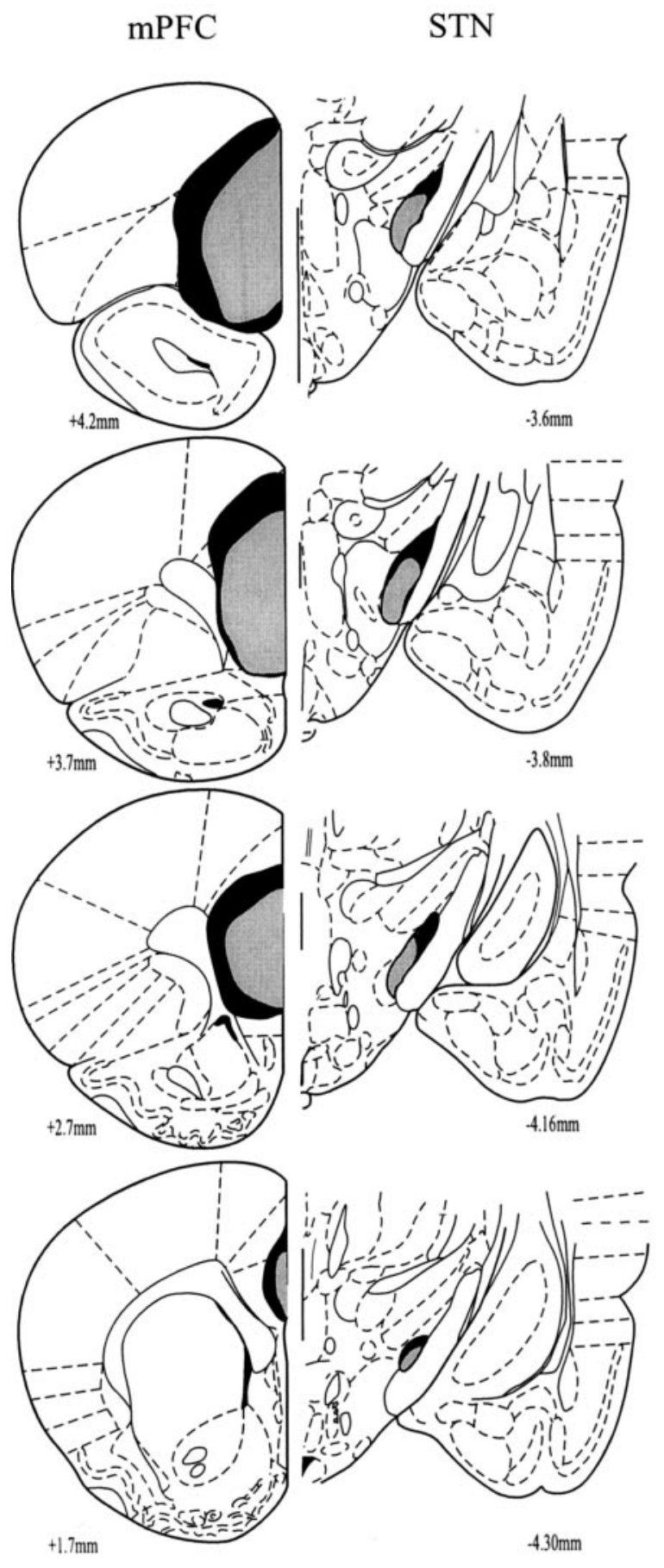

Figure 1. Diagrammatic reconstruction of coronal sections of rat brain showing the largest (black) and smallest (gray) extent of mPFC (left hemisphere) and STN (right hemisphere) lesions in the disconnected lesioned group. The numbers indicate AP coordinates relative to bregma. Atlas plates were adapted from Paxinos and Watson (1997).

for the unilateral groups were as follows: sham, $n=8$; unilateral mPFC, $n=6$; unilateral STN, $n=6$.

\section{Behavioral results}

Preoperative analysis

Before surgery, the groups of animals were well matched on all measures. No significant differences were observed in performance accuracy (mean, 85.2\% correct), omissions (mean, 6.5\%), premature responding (mean, 9), perseverative responding 
(mean, 29), correct latency (mean, $53 \mathrm{csec}$ ), magazine latency (mean, $143 \mathrm{csec})$, and panel pushes (mean, 55) $\left(F_{(1,23)} ; p>0.05\right)$.

\section{Baseline sessions}

Preliminary analysis of the sham data revealed that there was no significant difference between the two sham groups (sham-IPL and sham-DISC) in terms of performance accuracy across the 12 postoperative baseline sessions $\left(F_{(1,6)}=0.01 ; p>0.05\right)$. Thus, all of these animals were treated as a single sham group for subsequent analyses.

Relative to the sham controls and the IPL group, animals with DISC lesions showed profound impairments in all behavioral measures. These animals were markedly impaired in their ability to respond correctly to the visual target $\left(F_{(2,23)}=21.3 ; p<0.001\right)$ (Fig. 2A). This impairment was accompanied by an increase in the number of errors of omission $\left(F_{(2,23)}=14.8 ; p<0.001\right)$, (means: shams, 6.5\%; IPL, 14\%; DISC, 27\%). All animals showed a reduction in the number of omissions over sessions $\left(F_{(8,187)}=\right.$ 7.16; $p<0.001)$, and a significant session by lesion interaction was observed $\left(F_{(16,187)}=2.25 ; p<0.01\right)$. In addition, although all animals postoperatively were slower to make a correct response $\left(F_{(2,48)}=4.3 ; p<0.01\right)$, the DISC group showed significantly slower latencies compared with the other groups (Tukey's $p<$ $0.001 ; F_{(2,23)}=24.6 ; p<0.001$ ) (Fig. $2 B$ ). These animals also showed significantly longer magazine latencies $\left(F_{(2,23)}=3.86\right.$; $p<0.05)$ that recovered over sessions $\left(F_{(2,48)}=4.3 ; p<0.01\right)$.

ANOVA showed that although all animals showed a reduction in premature responding over sessions $\left(F_{(7,164)}=2.15 ; p<0.05\right)$, animals with DISC lesions made significantly more premature responses relative to the sham and IPL groups (Tukey's $p<0.001$; $F_{(2,23)}=17.56 ; p<0.001$ ) (Fig. $2 C$ ). In addition, the group with IPL lesions also showed a tendency to make more premature responses compared with shams (Tukey's $p=0.06$ ). Figure $2 D$ shows that the DISC-lesioned animals were also highly perseverative (Tukey's $p<0.001 ; F_{(2,23)}=7.22 ; p<0.01$ ) relative to the other groups. This effect remained stable over time, because there was no effect of session $\left(F_{(1,45)}=0.77 ; p>0.05\right)$ or a significant lesion by session interaction $(F<1)$. Repeated-measures ANOVA showed an overall reduction in perseverative panel pushes with increasing session $\left(F_{(5,134)}=2.43\right.$; $\left.p<0.05\right)$. However, despite showing an increase in perseverative panel pushes (means: shams, 51; IPL, 100; DISC, 114), the IPL and DISC groups were not significantly different from the sham-operated controls $\left(F_{(2,23)}=1.05 ; p>0.05\right)$.

To summarize, animals with lesions made to the STN and $\mathrm{mPFC}$ in contralateral hemispheres were significantly impaired in terms of discriminative accuracy. Post hoc tests confirmed that animals with lesions made in the ipsilateral hemisphere were not significantly different from sham controls. This discriminative impairment was accompanied by long latencies in making correct responses and collecting food reward. In addition, the disconnected animals were particularly impaired in withholding additional inappropriate nosepoke responses, as shown by significant increases in perseverative and premature responses. However, there were no effects on perseverative panel pushes at the magazine.

\section{Increased stimulus duration}

In an attempt to reduce the attentional load for the DISClesioned group and therefore facilitate an accurate percentage of correct responding in these animals, the duration of the stimulus was increased from the standard $0.5 \mathrm{sec}$ to 1,2 , and $4 \mathrm{sec}$. Unfortunately, data for nine animals at the $2 \mathrm{sec}$ stimulus duration (three sham animals, four IPL animals, and two DISC animals) were lost because of a computer failure. Analysis for this manipulation was based on the remaining animals for that group (five shams, three IPL animals, and eight DISC animals).

Figure $3 A$ shows the performance accuracy of all three groups when the stimulus duration was increased. Animals with DISC lesions showed improved performance with increased duration $\left(F_{(3,33)}=9.13 ; p<0.001\right)$, although they remained significantly impaired at all durations relative to the sham group (Tukey's $p<$ $\left.0.01 ; F_{(2,13)}=4.55 ; p<0.01\right)$ but were no different from the IPL group (Tukey's $F<1$ ). There was no lesion by stimulus duration interaction $\left(F_{(5,33)}=1.08 ; p>0.05\right)$.

In parallel with the effect on discriminative accuracy, the percentage of omissions decreased in all groups $\left(F_{(2,25)}=4.81 ; p<\right.$ $0.01)$, although the DISC-lesioned animals made significantly more errors of omission than the sham controls but not the IPL group (Tukey's $\left.p<0.05 ; F_{(2,13)}=4.88 ; p<0.05\right)$. No interaction was observed on this measure $(F<1)$.

Increasing the stimulus duration significantly lengthened the latency to make a correct response in the DISC-lesioned group $\left(F_{(2,13)}=8.77 ; p<0.01\right)$ (Fig. $\left.3 B\right)$. However, although animals with IPL lesions were no different from sham controls on this measure, they showed a tendency to respond more slowly (Tukey's $p=0.07)$. In marked contrast, no main effect of lesion was found for magazine latency $\left(F_{(2,13)}=2.78 ; p>0.05\right)$.

Figure $3 C$ shows how increasing the stimulus duration reduced the number of premature responses for all groups $\left(F_{(2,13)}=1.93 ; p>0.05\right)$. However, the DISC group maintained a high level of perseverative responding across all stimulus durations relative to sham controls (Tukey's $p<0.05 ; F_{(2,13)}=5.01$; $p<0.05$ ) but was not different from the sham or IPL groups in terms of perseverative panel pushes $\left(F_{(2,13)}=1.13 ; p>0.05\right)$. No other effects were observed for this measure.

To summarize, lengthening the stimulus duration improved accurate performance in the disconnected group, although these animals remained significantly impaired in accuracy when compared with other groups. In addition, increasing the stimulus duration failed to abolish all other deficits, including omissions, response latencies, and perseverative responses observed in the disconnected group, and did not affect the latency to collect reward pellets.

\section{Behavioral recovery: permanent and transient effects}

Figure 2 shows the behavior of animals during the last five baseline sessions (session numbers: 17, 19, 21, 23, 25). These sessions occurred toward the end of the postoperative testing period and were analyzed to provide some indication of the extent of recovery attained over time. Analysis of these sessions indicated that the DISC group maintained a significant impairment in performance accuracy (Tukey's $p<0.01 ; F_{(2,21)}=16.1 ; p<0.001$ ) throughout baseline testing that was accompanied by an increase in the number of omissions relative to sham controls and the IPL group (Tukey's $p<0.01 ; F_{(2,22)}=14.2 ; p<0.001$ ). The IPL group also made more omissions compared with shams, but was not different from the DISC-lesioned group (Tukey's $p<0.05$ ). This measure also revealed a baseline session by lesion interaction $\left(F_{(8,88)}=2.69 ; p<0.01\right)$. Unlike the postoperative testing schedule, there was no effect of lesion on premature responses $\left(F_{(2,22)}=2.53 ; p>0.05\right)$, although a significant lesion by baseline session interaction was obtained, as revealed by animals of the DISC group showing an increase in premature responding primarily during sessions 19 and 23 compared with sham animals 
A

Percent accuracy
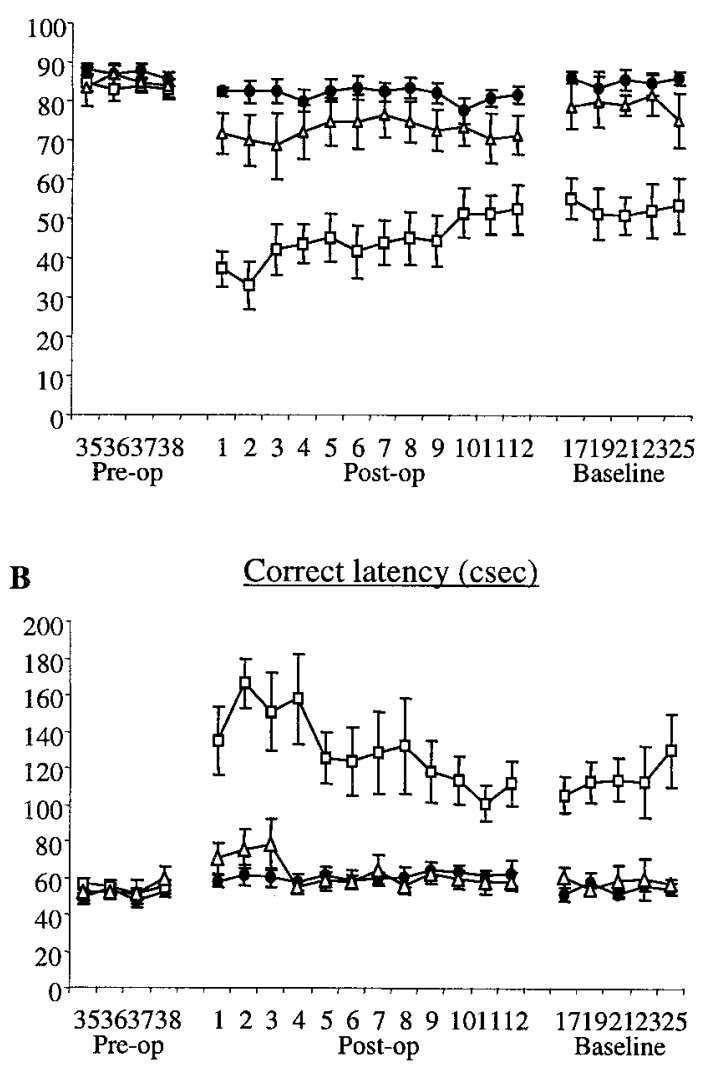

C

Premature responses
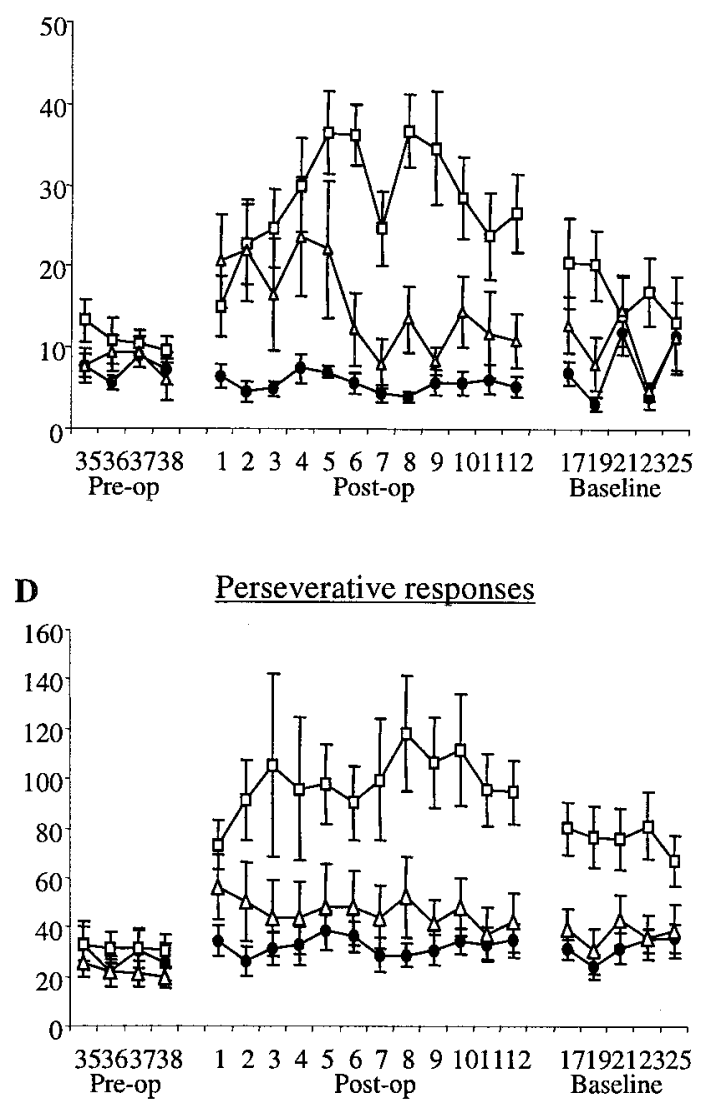
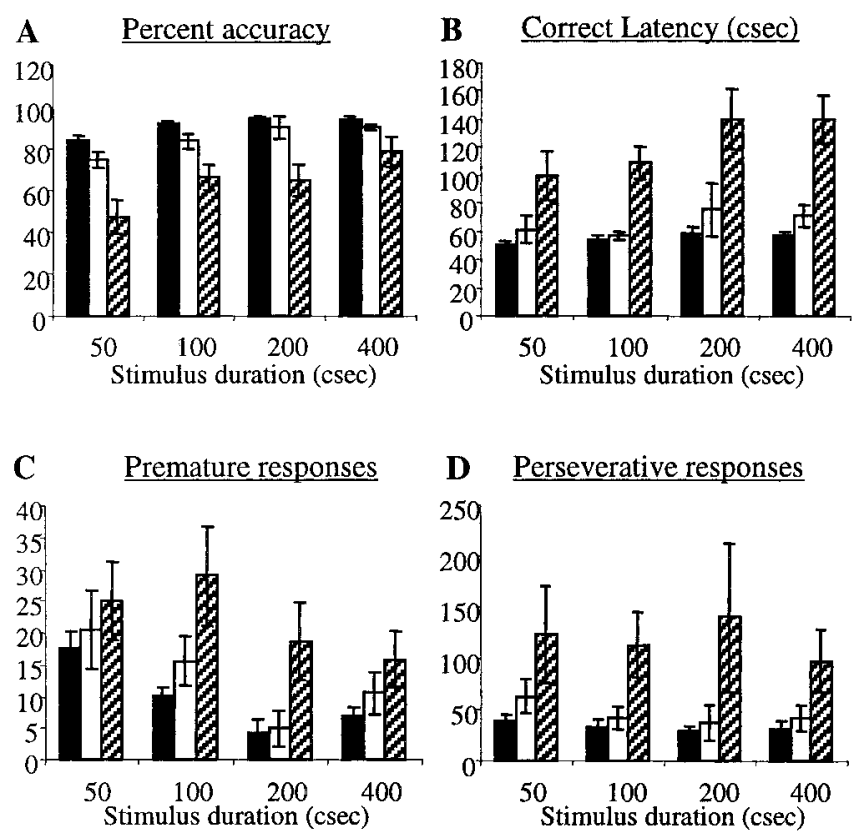

Figure 3. Effects of manipulating the stimulus duration on task performance of sham controls (closed bars), the IPL group (open bars), and the DISC group (hatched bars). Each bar represents mean \pm SEM. $A$, Percentage of accuracy. $B$, Correct latency (in centiseconds). $C$, Premature responses. $D$, Perseverative responses. Each stimulus duration was presented in a single test session.

and the IPL group $(p<0.01)$. There were no lesion differences in premature responding during session $25(p>0.05)$.

Animals with DISC lesions continued to make significantly more perseverative responses relative to the sham and IPL groups during the baseline sessions (Tukey's $p<0.01 ; F_{(2,21)}=10.63$; $p<0.001)$, but these animals did not differ in terms of the number of perseverative panel pushes $(p>0.05)$. These animals also maintained significantly long latencies when making the response (Tukey's $\left.p<0.01 ; F_{(2,22)}=19.05 ; p<0.001\right)$ and when collecting their reward pellet (Tukey's $p<0.01 ; F_{(2,20)}=10.98$; $p<0.001)$. No other effects were observed.

In summary, disconnected animals maintained profound behavioral deficits in discriminative accuracy, number of omissions, perseverative responses, and overall speed, indicating a lack of behavioral recovery over time for these measures. In contrast, the level of premature responding decreased over time postoperatively, suggesting a more transient effect of these lesions on this measure.

Effects of single unilateral STN or unilateral mPFC lesions on postoperative baseline performance

As illustrated in Figure 4A, the mean performance accuracy was significantly lower in animals with a single unilateral mPFC or unilateral STN lesion $\left(F_{(2,17)}=6.23 ; p<0.01\right)$, because both lesioned groups were significantly different from shams (Tukey's $p<0.01)$. Relative to the DISC group, all animals improved accuracy $\left(F_{(1,31)}=7.71 ; p<0.01\right)$ and reduced the number of

Figure 2. Effects of theDISC group (open squares), IPL group (open triangles), and sham group (closed circles) on performance of the standard baseline task on the 5CSRTT. A, Percentage of accuracy. $B$, Correct latency (in centiseconds). C, Premature responses. D, Perseverative responses. Mean \pm SEM results are illustrated for the last four sessions of preoperative baseline (Pre-op), $12 \mathrm{~d}$ of postoperative testing (Post-op), and five additional baseline sessions (Baseline). 


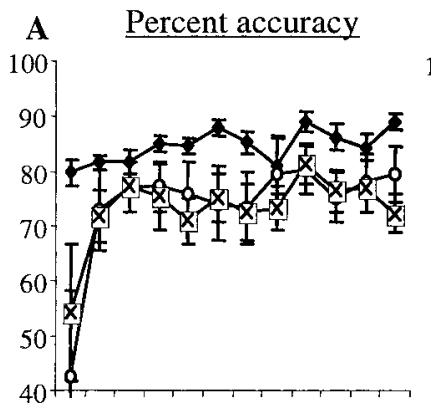
Session
1233456789101112

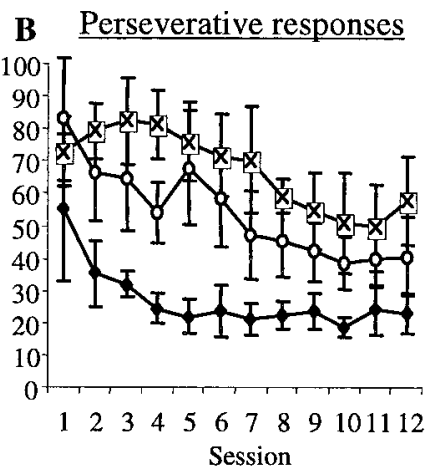

Figure 4. Mean \pm SEM performance of sham controls (closed diamonds) and unilateral $\mathrm{mPFC}$ (open circles) and unilateral STN $(X)$ lesion groups on $12 \mathrm{~d}$ of postoperative baseline on the 5CSRTT. A, Percentage of accuracy. $B$, Perseverative responses.

omissions $\left(F_{(1,65)}=5.92 ; p<0.001\right)$ over sessions. However, animals with unilateral STN lesions made significantly more errors of omission (Tukey's $p<0.05 ; F_{(2,17)}=3.84 ; p<0.05$ ) compared with the unilateral $\mathrm{mPFC}$ group or sham controls (data not shown).

The number of premature responses declined in all groups as the number of sessions increased $\left(F_{(1,146)}=4.92 ; p<0.001\right)$, but there was no effect of lesion group on premature responding $(p>0.05)$. In contrast, unilateral lesions made to the mPFC or the STN produced a marked increase in perseverative responses relative to sham controls (Tukey's $p>0.05 ; F_{(2,17)}=8.23 ; p<$ 0.01 ) (Fig. 4B). The unilateral mPFC or unilateral STN were not different from each other (means: sham, 27; unilateral mPFC, 54; unilateral STN, 67). Although all animals showed a relative reduction in perseverative panel pushes, there were no main effects of lesion on this measure $\left(F_{(2,17)}=0.03 ; p>0.05\right)$.

Although animals with unilateral STN lesions were slower at making a correct response relative to the unilateral mPFC group or the sham group (Tukey's $p>0.01 ; F_{(2,17)}=5.57 ; p<0.01$ ), they were no different in terms of magazine latency $\left(F_{(2,17)}=\right.$ $0.07 ; p>0.05)$. No other effects were observed.

In summary, animals with single unilateral lesions of the STN or mPFC were impaired in performance accuracy and perseverative responding but not premature responding. Only the unilateral STN group showed increased omissions and longer latencies to respond, whereas magazine latency was unaffected in all animals.

\section{Summary comparisons among bilaterally lesioned and unilaterally lesioned groups}

For the 12 postoperative baseline sessions, the data were collapsed across session and analyzed to make overall behavioral comparisons among the groups. There were no significant differences between the sham group used in the disconnection study analysis and the sham group used for the unilateral lesion analysis $(F<1)$. Figure 5 illustrates the mean differences between groups. The pattern of impairments was qualitatively similar for accuracy, perseveration, and latency measures except for premature responding. The DISC group was significantly impaired in discriminative accuracy compared with all groups (Tukey's $p<$ $\left.0.01 ; F_{(4,45)}=21.49 ; p<0.001\right)$, whereas the IPL group was no different from the unilateral groups or sham controls. An effect of lesion was also obtained for a number of omissions $\left(F_{(4,45)}=\right.$ 7.87; $p<0.001$ ), which was attributable primarily to the DISC group being different from all groups (Tukey's $p<0.05$ ) except the unilateral STN group. In comparison, animals with IPL were

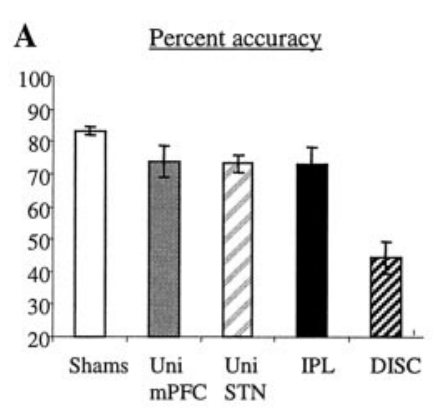

B Correct latency (csec)
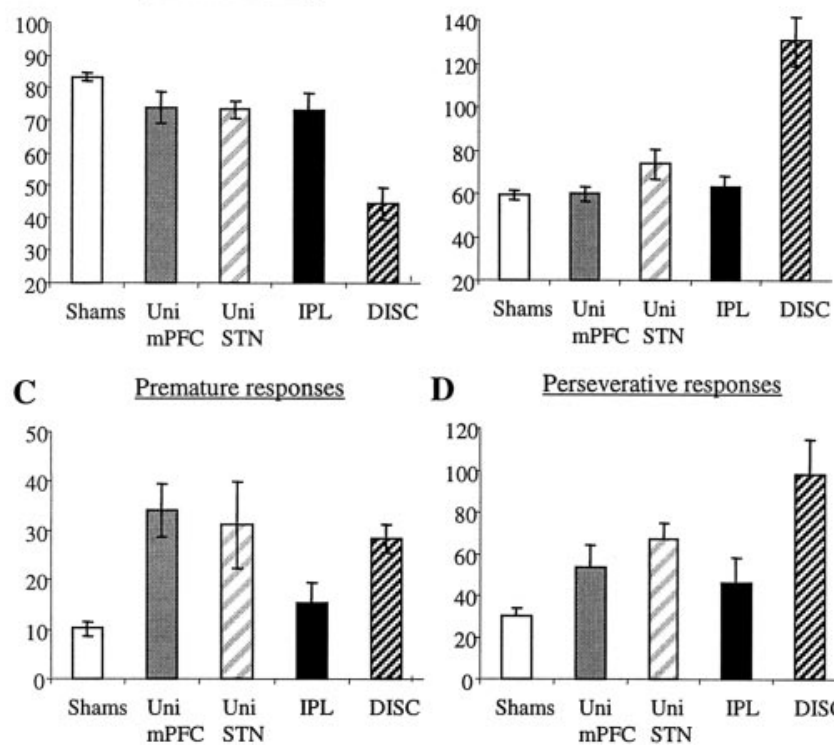

D Perseverative responses

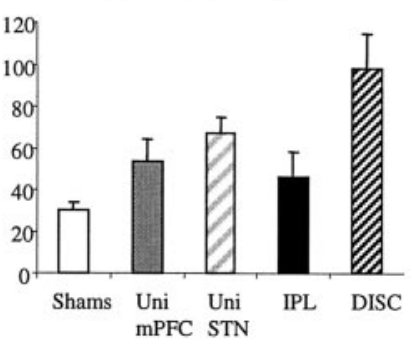

Figure 5. Summary comparisons among groups for 12 postoperative baseline sessions: Sham group, open bars; unilateral mPFC (Uni mPFC) group, gray bars; unilateral STN (Uni STN) group, thick-hatched bars; IPL, closed bars; DISC group, narrow-hatched bars. Data were collapsed across session, and mean data \pm SEM are presented. $A$, Percentage of accuracy. $B$, Correct latency (in centiseconds). C, Premature responses. D, Perseverative responses.

different only from shams but were not different from either of the unilateral groups. Similarly, relative to all other groups, the DISC group was slower in response latency (Tukey's $p<0.001$; $\left.F_{(4,44)}=26.02 ; p<0.001\right)$ and magazine latency (Tukey's $p<$ $\left.0.01 ; F_{(4,44)}=6.62 ; p<0.001\right)$. No other differences were observed among the other groups.

There was a significant difference of lesion on premature responding $\left(F_{(4,45)}=9.73 ; p<0.001\right)$. This difference was attributable primarily to the IPL group making fewer premature responses compared with the unilateral mPFC group (Tukey's $p<$ 0.01 ; means: IPL, 15; unilateral mPFC, 33) and the unilateral STN group ( $p=0.06$; means: IPL, 15 ; unilateral STN, 31 ). The IPL group was found not to be different from the DISC group ( $p=$ 0.08; means: IPL, 15; DISC, 28) (Fig. 5C). The IPL group was not different from the sham controls on this measure (means: sham, 10; IPL, 15).

Overall, there was an effect of lesion on perseverative responses that was attributable to the DISC group being more perseverative relative to all groups (Tukey's $p<0.05 ; F_{(4,44)}=6.62$; $p<0.001$ ) (Fig. 5D). However, the IPL group, although making fewer perseverative responses compared with DISC animals, was not different from the unilateral groups or sham controls. No differences were observed for perseverative panel pushes $(F<1)$.

In summary, the disconnected lesioned animals were less accurate in their performance, slower to respond, and highly perseverative. In comparison, the IPL groups performed at the same level as animals with unilateral lesions and controls. However, the IPL lesion made significantly fewer premature responses compared with either the unilateral or the disconnected groups and was not different from the sham controls.

\section{Locomotor activity}

One-way ANOVA showed that animals with DISC lesions were not different in spontaneous locomotor activity compared with sham controls or the IPL group $\left(F_{(2,22)}=2.13 ; p>0.05\right)$, sug- 
gesting that the deficits in response control observed in this group were not a function of general hyperactivity.

\section{Discussion}

This is the first study to demonstrate the functional interaction and conjoint importance of the $\mathrm{MPFC}$ and the STN in attentional performance. Animals with corticosubthalamic disconnections showed profound attentional impairments, were highly perseverative, and had slowed response latencies. These behavioral deficits were greater and more persistent than those found after unilateral lesions of either structure alone and were comparable with some of the effects of bilateral lesions of either the STN (Baunez and Robbins, 1997, 1999a) or the mPFC (Muir et al., 1996; Passetti et al., 2000). The ipsilateral group, in which the combined lesions of the MPFC and STN were restricted to one hemisphere only, showed deficits that were strikingly different from the disconnected group in that they were transient and minor. The IPL group not only provided a control for a nonspecific "mass-action" effect of the dual lesion but also signified the relative contributions of the mPFC and STN in an anatomical circuit that was compromised only when the critical pathway between these structures was damaged in both hemispheres.

\section{Behavioral nature of the deficits}

The severe deficit in accuracy induced by the DISC lesions accompanied by the increased incidence in omissions indicates a pronounced attentional impairment. When the attentional load was reduced by gradually increasing the stimulus duration, the DISC group showed a progressive increase in accuracy, thus ruling out the possibility that these animals were impaired in their ability to correctly perceive the target stimulus or undermotivated to perform the task. The partial recovery in these animals also suggests that the discriminative impairment is unlikely to be a consequence of failing to make associations or indeed failures in remembering where the stimuli were presented. Effects of interference can also be ruled out, because these animals did not perseverate at the panel that would otherwise have prevented them from correctly orienting toward the visual target. Nonetheless, increasing the stimulus duration to $4 \mathrm{sec}$ failed to completely alleviate the accuracy impairment in the DISC group, suggesting the animal's inability to scan and monitor the visual array. The omission rate may have been related to the increased response latencies that were observed in the DISC group, suggesting an overall deficit in decisional processes (Baunez et al., 2001).

The impairment observed in DISC lesions was not restricted to attention. The increase in perseverative responding persisted even when increasing the stimulus duration helped to alleviate the accuracy deficit. This suggests that the deficit in perseveration was relatively independent of the processes involving stimulus detection, reflecting an inability to suppress irrelevant responses. At the same time, however, the DISC group showed only a transient impairment in premature responding, which recovered over time. This implies that the disinhibitory mechanisms impaired in perseveration may be different from those involved in anticipatory, premature behavior. In addition, whereas bilateral $\mathrm{mPFC}$ lesions enhanced perseverative nose pokes without affecting premature responding, lesions of the postgenual anterior cingulate cortex produced increased premature but not perseverative responding (Muir et al., 1996). Clearly, this double dissociation indicates two types of inhibitory processes that can be differentiated at the level of the frontal cortex. The finding that bilateral STN lesions produce both perseverative and premature deficits implies that the STN is indeed involved in the executive control of responding (Baunez and Robbins, 1997), although its behavioral expression is likely influenced by the cortical origin of its projection. This hypothesis needs to be tested directly.

Together, our results confirm the involvement of a selective corticosubthalamic interaction in discrimination and the executive control of responding. The overall outcome (i.e., double IPL lesions failed to exhibit deficits greater than those seen after single unilateral lesions) is consistent with the present disconnection results. However, the interesting finding that the IPL lesion exhibited a level of premature responding that was no different from shams but also lower than the unilateral and DISC groups suggests that the STN and mPFC may independently mediate such premature, impulsive behaviors via neural mechanisms other than the direct corticosubthalamic projection, especially given that bilateral STN lesions increase premature responses (Baunez and Robbins, 1997). One speculation is that selective regions of the $\mathrm{MPFC}$ promote different levels of disinhibition (Y. Chudasama and T. W. Robbins, unpublished observations).

Functional organization of the corticosubthalamic projection The functional organization of the basal ganglia identifies the STN as a relay by which corticostriatal information is transmitted to the output nuclei, resulting in some modulation of behavior (Albin et al., 1989; DeLong, 1990; Shink et al., 1996). In line with this model, parallel studies have shown that corticostriatal disconnections (Christakou et al., 2001), bilateral striatal lesions (Rogers et al., 2001), and bilateral STN lesions (Baunez and Robbins, 1997; Baunez et al., 2001) affect aspects of motivated behavior as well as response selection and control. However, increasing anatomical and physiological evidence (Bolam et al., 2002) supports a revised model that places a particular emphasis on the corticosubthalamic projection.

Our data suggest that the corticosubthalamic pathway is a route by which the cortex can influence the activity of the STN and integrate behavior relatively independently from the corticostriatal interaction, while profoundly influencing the functional properties of the entire indirect pathway. Hence, a disruption of the cortical influence on the STN may help explain the impairments observed in both the DISC and unilateral groups. When either the MPFC or STN is inactivated, hypothetically, the excitatory signal from the cortex to the STN is reduced. The STN is also disinhibited by the GABAergic neurons of the globus pallidus, to which the STN is reciprocally connected. The feedback from the STN to the globus pallidus is important, because neurons within this structure do not fire bursts of action potentials if the STN is already exhibiting burst firing (Magill et al., 2000). In addition, neurons of the globus pallidus also provide powerful inhibitory feedback to the striatum (Magill et al., 2000). These lines of evidence suggest that the firing pattern in the STN must be provided by the corticosubthalamic pathway, and that the corticosubthalamic pathway is critical in determining the activity of the globus pallidus (Magill et al., 2000; Bolam et al., 2002). Furthermore, the striatum, because of its feedback from the globus pallidus, may modify cortical flow through the basal ganglia (Ryan and Clark, 1991; Bolam et al., 2002). Additionally, the STN provides a major excitatory drive on output structures such as the substantia nigra pars reticulata (SNpr) (Hammond et al., 1978; Nakanishi et al., 1987). Thus, depriving the STN of its excitatory glutamatergic input from the cortex may result in decreased excitation of the SNpr, which is also under an inhibitory striatonigral projection (Nakanishi et al., 1987). Decreased nigral activity is thought to disinhibit the motor thalamus and, therefore, result in hyperkinetic movements and other related deficits observed 
after STN lesions (DeLong, 1990). Furthermore, inactivating the STN by manipulating its glutamatergic and GABAergic afferents (to mimick either a blockade of the cortical inputs or a reinforcing inhibition from the globus pallidus) replicates the marked deficits on the 5CSRTT normally produced by STN lesions alone (Baunez and Robbins, 1999b), suggesting that changes in the STN are not related exclusively to globus pallidus activity. Finally, both the $\mathrm{MPFC}$ and STN receive excitatory dopaminergic projections from the substantia nigra and ventral tegmental area (Lindvall and Björklund, 1983; Canteras et al., 1990), and it has been suggested recently that under certain conditions (e.g., striatal dopamine depletion), the neurons of the STN, while exhibiting abnormal bursting activity, may essentially distort the processing of cortical information (Bergman et al., 1994; Magill et al., 2001; Bolam et al., 2002).

\section{Functional implications}

This study is the first to demonstrate that a corticosubthalamic disconnection induces a behavioral syndrome of discriminative and perseverative deficits in a continuous performance type of attentional task. By implication, the "intact" corticosubthalamic projection may function to integrate complex behaviors that require the selection, suppression, and execution of a planned and controlled sequence of responses that mediate efficient task performance (Robbins, 1996). Such executive control deficits are a hallmark of frontal lobe patients, who are often described as lacking normal executive control over action, as shown by their poor performance in tests of planning (Shallice, 1982). There is now accumulating evidence that patients with Parkinson's disease show cognitive deficits that resemble those observed in frontal patients (Morris et al., 1988; Owen et al., 1990, 1992; Cools et al., 2002). However, Parkinson's disease is thought to result primarily from the dysfunction of the frontostriatal circuit and its dopaminergic modulation. The role of the STN in Parkinson's disease has been considered with respect to motor disturbances (Bergman et al., 1990; Limousin et al., 1995), but there is little evidence that implicates the dysfunction of a direct corticosubthalamic projection. The recent findings, however, that deep brain stimulation (which is thought to inactivate the STN) improves motor deficits in Parkinson's disease while, at the same time, producing marked executive impairments (Jahanshahi et al., 2000; Saint-Cyr et al., 2000; Schroeder et al., 2002), suggests that it may potentially modify effective frontal processing by disrupting the corticosubthalamic projection. The findings from this study suggest that the function of the STN is critically dependent on an intact frontal cortex, and that the frontal cortex may exert significant control of behavior at the level of poststriatal processing.

\section{References}

Afsharpour S (1985) Topographical projections of the cerebral cortex to the subthalamic nucleus. J Comp Neurol 236:14-28.

Albin RL, Young AB, Penny JB (1989) The functional anatomy of basal ganglia disorders. Trends Neurosci 12:366-375.

Baunez C, Robbins TW (1997) Bilateral lesions of the subthalamic nucleus induce multiple deficits in an attentional task in rats. Eur J Neurosci 9:2086-2099.

Baunez C, Robbins TW (1999a) Effects of dopamine depletion of the dorsal striatum and further interaction with subthalamic nucleus lesions in an attentional task in the rat. Neuroscience 92:1343-1356.

Baunez C, Robbins TW (1999b) Effects of transient inactivation of the subthalamic nucleus by local muscimol and APV infusions on performance on the five-choice serial reaction time task in rats. Psychopharmacology (Berl) 141:57-65.
Baunez C, Nieoullon A, Amalric M (1995) In a rat model of parkinsonism, lesions of the subthalamic nucleus reverse increases of reaction time, but induce a dramatic premature responding deficit. J Neurosci 15:6531-6541.

Baunez C, Humby T, Eagle DM, Ryan LJ, Dunnett SB, Robbins TW (2001) Effects of STN lesions on simple vs choice reaction time tasks in the rat: preserved motor readiness, but impaired response selection. Eur J Neurosci 13:1609-1616.

Berendse HW, Groenewegen HJ (1991) The connections of the medial part of the subthalamic nucleus in the rat: evidence for a parallel organisation. In: The basal ganglia III (Bernardi G, Carpenter MB, DiChiara G, Morelli M, Stanzione P, eds), pp 89-98. New York: Plenum.

Bergman H, Wichmann T, Delong MR (1990) Reversal of experimental parkinsonism by lesions of the subthalamic nucleus. Science 249:1436-1438.

Bergman H, Wichmann T, Karmon B, Delong MR (1994) The primate subthalamic nucleus. II. Neuronal activity in the MPTP model of Parkinsonism. J Neurophysiol 72:507-520.

Berns GS, Sejnowski TJ (1996) How the basal ganglia make decisions. In: Neurobiology of decision making (Damasio A, Damasio H, Christen Y, eds), pp 101-113. Berlin: Springer.

Bolam JP, Magill JP, Bevan MD (2002) The functional organization of the basal ganglia: new insights from anatomical and physiological analyses. In: The basal ganglia VII (Nicholson LFB, Faull RLN, eds), pp 371-378. New York: Plenum.

Canteras NS, Shammah-Lagnado SJ, Silva BA, Ricardo JA (1990) Afferent connections of the subthalamic nucleus: a combined retrograde and anterograde horseradish peroxidase study in the rat. Brain Res 513:43-59.

Carli M, Robbins TW, Evenden JL, Everitt BJ (1983) Effects of lesions to ascending noradrenergic neurones on performance of a 5-choice serial reaction task in rats: implications for theories of dorsal noradrenergic bundle function based on selective attention and arousal. Behav Brain Res 9:361-380.

Christakou A, Robbins TW, Everitt BJ (2001) Functional disconnection of a prefrontal cortical-dorsal striatal system disrupts choice reaction time performance: implications for attentional function. Behav Neurosci 115:812-825.

Cools R, Stefanova E, Barker RA, Robbins TW, Owen AM (2002) Dopaminergic modulation of high-level cognition in Parkinson's disease: the role of the prefrontal cortex revealed by PET. Brain 125:584-594.

DeLong MR (1990) Primate models of movement disorders of basal ganglia origin. Trends Neurosci 13:281-285.

Fujimoto K, Kita H (1993) Response characteristics of subthalamic neurons to the stimulation of the sensorimotor cortex in the rat. Brain Res 609:185-192.

Hammond C, Deniau JM, Rizk A, Féger J (1978) Electrophysiological demonstration of an excitatory subthalamonigral pathway in the rat. Brain Res 151:235-244.

Jahanshahi M, Ardouin CM, Brown RG, Rothwell JC, Obeso J, Albanese A, Rodriguez-Oroz MC, Moro E, Benabid AL, Pollak P, Limousin-Dowsey P (2000) The impact of deep brain stimulation on executive function in Parkinson's disease. Brain 123:1142-1154.

Kitai ST, Deniau JM (1981) Cortical inputs to the subthalamic nucleus: intracellular analysis. Brain Res 214:411-415.

Levy R, Hazrati LN, Herrero MT, Vila M, Hassani OK, Mouroux M, Ruberg M, Asensi H, Agid Y, Féger J, Obeso JA, Parent A, Hirsch EC (1997) Re-evaluation of the functional anatomy of the basal ganglia in normal and Parkinsonian states. Neuroscience 76:333-343.

Limousin P, Pollak P, Benazzouz A, Hoffmann D, Le Bas JF, Broussolle E, Perret JE, Benabid AL (1995) Effect on parkinsonian signs and symptoms of bilateral subthalamic nucleus stimulation. Lancet 345:91-95.

Lindvall O, Björklund A (1983) Dopamine- and norepinephrine-containing neuron systems: their anatomy in the rat brain. In: Chemical neuroanatomy (Emson PC, ed), pp 229-255. New York: Raven.

Magill PJ, Bolam JP, Bevan MD (2000) Relationship of activity in the subthalamic nucleus-globus pallidus network to cortical electroencephalogram. J Neurosci 20:820-833.

Magill PJ, Bolam JP, Bevan MD (2001) Dopamine regulates the impact of the cerebral cortex on the subthalamic nucleus-globus pallidus network. Neuroscience 106:313-330.

Maurice N, Deniau JM, Glowinski J, Thierry AM (1998) Relationships be- 
tween the prefrontal cortex and the basal ganglia in the rat: physiology of the corticosubthalamic circuits. J Neurosci 18:9539-9546.

Morris RG, Downes JJ, Sahakian BJ, Evenden JL, Heald A, Robbins TW (1988) Planning and spatial working memory in Parkinson's disease. J Neurol Neurosurg Psychiatry 51:757-766.

Muir JL, Everitt BJ, Robbins TW (1996) The cerebral cortex of the rat and visual attentional function: dissociable effects of mediofrontal, cingulate, anterior, dorsolateral and parietal cortex lesions on a five choice serial reaction time task. Cereb Cortex 6:470-481.

Nakanishi H, Kita H, Kitai ST (1987) Intracellular study of rat substantia nigra pars reticulata neurons in an in vitro slice preparation: electrical membrane properties and response characteristics to subthalamic stimulation. Brain Res 437:45-55.

Owen AM, Downes JJ, Sahakian BJ, Polkey CE, Robbins TW (1990) Planning and spatial working memory following frontal lobe lesions in man. Neuropsychologia 28:1021-1034.

Owen AM, James M, Leigh PN, Summers BA, Marsden CD, Quinn NP, Lange KW, Robbins TW (1992) Fronto-striatal cognitive deficits at different stages of Parkinson's disease. Brain 115:1727-1751.

Parent A, Hazrati LN (1995) Functional anatomy of the basal ganglia. II. The place of subthalamic nucleus and external pallidum in basal ganglia circuitry. Brain Res Brain Res Rev 20:128-154.

Passetti F, Humby T, Everitt BJ, Robbins TW (2000) Mixed attentional and executive deficits in medial frontal cortex lesioned rats. Psychobiology 28:261-271.

Paxinos G, Watson C (1997) The rat brain in stereotaxic coordinates, Ed 2. Sydney: Academic.
Robbins TW (1996) Dissociating executive functions of the prefrontal cortex. Philos Trans R Soc Lond B Biol Sci 351:1463-1470.

Rogers RD, Baunez C, Everitt BJ, Robbins TW (2001) Lesions of the medial and lateral striatum in the rat produce differential deficits in attentional performance. Behav Neurosci 115:799-811.

Rouzaire-Dubois B, Scarnati E (1985) Bilateral corticosubthalamic nucleus projections: an electrophysiological study in rats with chronic cerebral lesions. Neuroscience 15:69-79.

Ryan LJ, Clark KB (1991) The role of the subthalamic nucleus in the response of globus pallidus neurons to stimulation of the prelimbic and agranular frontal cortices in rats. Exp Brain Res 86:641-651.

Saint-Cyr JA, Trepanier LL, Kumar R, Lozano AM, Lang AE (2000) Neuropsychological consequences of chronic bilateral stimulation of the subthalamic nucleus in Parkinson's disease. Brain 123:2091-2108.

Schroeder U, Kuehler A, Haslinger B, Erhard P, Fogel W, Tronnier VM, Lange KW, Boecker H, Ceballos-Baumann AO (2002) Subthalamic nucleus stimulation affects striato-anterior cingulate cortex circuit in a response conflict task: a PET study. Brain 125:1995-2004.

Shallice T (1982) Specific impairments of planning. Philos Trans R Soc Lond B Biol Sci 298:199-209.

Shink E, Bevan MD, Bolam JP, Smith Y (1996) The subthalamic nucleus and the external pallidum: two tightly interconnected structures that control the output of the basal ganglia in the monkey. Neuroscience 73: 335-357.

Winer BJ (1971) Statistical principles in experimental design. New York: McGraw-Hill. 